\title{
Broadband Balun Circuits Composed of Impedance Transforming Directional Couplers and LH Transmission-Line Sections
}

\author{
J. Sorocki, I. Piekarz, K. Staszek, P. Kaminski, K. Wincza, and S. Gruszczynski
}

\begin{abstract}
In this paper, a new approach to the design of a balun circuit is presented. The proposed balun is composed of an impedance-transforming directional coupler and an LH/RH transmission line differential phase shifter. The proposed solution features broadband amplitude and phase response, moreover, a flexibility of the impedance transformation from balanced to imbalanced port is obtained. The proposed concept has been verified by the design of a balun circuit featuring over-an-octave bandwidth and impedance transformation 70 $\Omega_{\text {bal. }} / 50 \Omega_{\text {imbal }}$.
\end{abstract}

Index Terms-Microwave baluns, directional couplers, coupled-line couplers.

\section{INTRODUCTION}

Baluns are well-known microwave circuits allowing for conversion of unbalanced mode propagating in asymmetric wave-guiding structures, e.g. microstrip lines, to a balanced one propagating in balanced lines. The design of such networks has been described in a number of papers [1]-[6]. Among typical solutions one can name tapered-line baluns [1] Marchand baluns [2]-[4] or baluns designed with the use of trans-directional couplers [5]. On the other hand a $0 / 180^{\circ}$ coupled-line directional coupler can be utilized as a balun circuit. As a benefit, a wide operational bandwidth can be obtained, taking the advantage of the broadband properties of the utilized coupled-line sections. The exemplary design of such networks can be found in e.g. [6], [7], where $0 / 180^{\circ}$ couplers have been designed as a connection of $3 \mathrm{~dB} / 90^{\circ}$ directional couplers and $90^{\circ}$ phase shifters. Moreover, in order to achieve wide operational bandwidth in terms of phase response, differential phase shifters utilizing left-handed (LH) and right-handed $(\mathrm{RH})$ transmission-line sections have been applied. In such networks, however, the impedance terminating the balanced port needs to be equal twice the impedance terminating the imbalanced port i.e. $Z_{\text {bal. }}=2 Z_{\text {imbal }}$. Therefore, the impedance transformation ratio defined as $R_{b}=$ $Z_{\text {bal. }} / Z_{\text {imbal }}$ equals 2 and cannot be arbitrarily chosen.

In this paper, a novel concept of the balun circuit has been proposed, in which recently developed

Manuscript received December 9, 2014; revised January 20, 2016. This work was partially supported by the National Science Centre under contract no. DEC-2011/01/D/ST7/00789 and in part by the statutory activity of AGH Department of Electronics.

The authors are with the Electronic Department, AGH University of Science and Technology, Av. Mickiewicza 30, 30-059 Krakow, Poland (Corresponding author: S. Gruszczynski; tel.: 0048-12-6173021; fax: 0048-12-633-23-98; e-mail: slawomir.gruszczynski@agh.edu.pl). impedance-transforming directional couplers have been applied. In the proposed network, the impedance transformation is obtained by the properly designed coupled-line section as it was recently reported [8]. The required phase response of the network is obtained with the use of an additional differential phase shifter composed of LH and RH transmission-line sections, as it was presented in [6] and [7]. As a result, broadband amplitude and phase response of the balun circuit can be obtained. The proposed technique has been verified by the design of a balun circuit transforming the impedance terminating imbalanced port $Z_{\text {imbal. }}=50 \Omega$ to the impedance terminating balanced port $Z_{b a l}=70 \Omega\left(R_{b}=\right.$ 1.4). The obtained measurement results are in a good agreement with the results of circuit and electromagnetic analyses.

\section{ANALYSIS OF THE PROPOSED BALUN CIRCUIT}

The concept of the proposed balun circuit is presented schematically in Fig. 1(a), where a circuit composed of a $90^{\circ}$-long coupled line section in conjunction with the ideal $90^{\circ}$ phase shifter is presented.

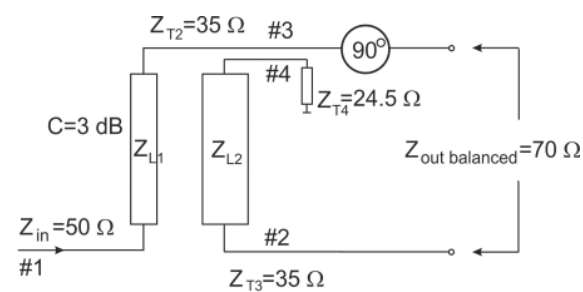

(a)

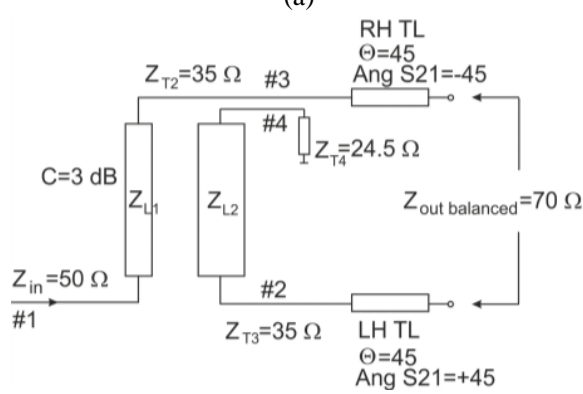

(b)

Fig. 1. Schematic diagram of the proposed balanced circuit utilizing an impedance transforming directional coupler. Balun with an ideal $90^{\circ}$ phase shifter (a) and with a differential phase shifter consisting of two $45^{\circ}$-long $\mathrm{LH}$ and RH transmission line sections (b).

If the coupled-line section is designed following the procedure shown in [8], the resulting coupled-line section transforms the impedance of the input port to the chosen impedance level at the coupled and transmission ports. As it 
was also shown in [8] the limiting value for the impedance transformation is related to the coupling factor $k$ of the coupled-line section, and is expressed as follows:

$$
R \leq \frac{1}{k^{2}}
$$

Taking into account that in baluns equal power split needs to be ensured i.e. $k=0.707$, the impedance transformation $R$ cannot exceed 2 . This condition, however, gives a flexibility for the realizable impedance transformation, which can be of any value from the range of $25 \Omega<Z_{\text {out }}<100 \Omega$ (assuming $Z_{\text {in }}$ $=50 \Omega$ ). The given range of output impedance of the coupled-line section $Z_{\text {out }}$ results in a range of realizable balanced impedance $50 \Omega<Z_{b a l}<200 \Omega$, that can be achieved at the balanced output ports of the circuit.

To investigate the properties of the proposed circuit, a balun with impedance transformation $R_{b}=Z_{\text {bal. }} / Z_{\text {imbal }}=70 \Omega$ / $50 \Omega$ has been designed, for which the coupling factor $k=$ 0.8 has been selected, ensuring a wide operational bandwidth in terms of amplitude response. The required additional $90^{\circ}$ differential phase shifter has been realized with the use of a technique reported in [6], [7], where a pair of LH and RH sections, both being $45^{\circ}$-long, have been applied (see Fig. 1(b). The resulting scattering parameters of the circuit are presented in Fig. 2.

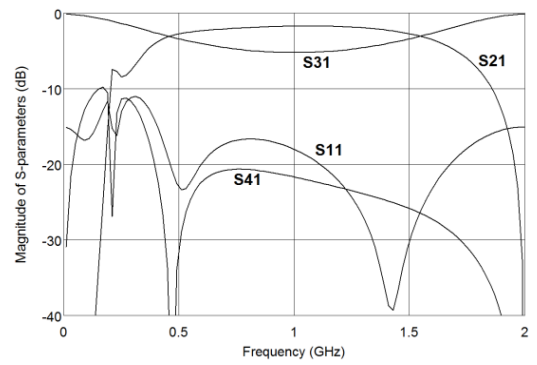

(a)

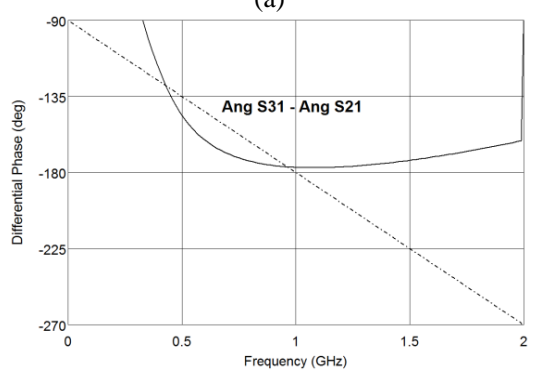

(b)

Fig. 2. Magnitude of $S$ parameters of the designed broadband balun circuit (a), and its differential phase (solid line) (b) in comparison to the differential phase of a balun with 'RH-only' phase shifter (dashed line). Results of circuit analysis.

The proposed circuit features wide operational bandwidth in terms of both amplitude and phase response. Additionally in Fig. 2(b) a phase response of the balun circuit is shown, in which the required $90^{\circ}$ phase shift is obtained using a $90^{\circ}$-long $\mathrm{RH}$ line at the transmission ports. From the comparison it is seen that the utilization of a metamaterial transmission line improves the phase imbalance in relatively wide frequency range. For the purpose of analysis the $45^{\circ}$-long LH transmission-line section has been approximated with the lumped element equivalent circuit presented in Fig. 3(a), for which the following values have been found $C=13$ $\mathrm{pF}$ and $L=16 \mathrm{nH}$. Further, to allow for physical realization, a semi-distributed approximation has been employed (see Fig. 3(b)) for which the following values have been found: $Z s=$ $170 \Omega, \Theta 1=32^{\circ}, \Theta 2=38^{\circ}, C=13 \mathrm{pF}$.

\section{BROADBAND BALUN CIRCUIT REALIZATION}

To experimentally verify the presented concept, a coupled line section has been designed in a coupled-line geometry shown in Fig. 4, for which $h_{1}=0.152 \mathrm{~mm}, \varepsilon_{r 1}=3.38, h_{2}=0.04$ $\mathrm{mm}, \varepsilon_{r 2}=3.38, h_{3}=1.524 \mathrm{~mm}, \varepsilon_{r 3}=3.38$ and $w_{1}=0.92 \mathrm{~mm}$, $w_{2}=3.71 \mathrm{~mm}$. The per-unit-length parameters, listed in Table I, and the dimensions of the strips have been found numerically using Linpar software [9].

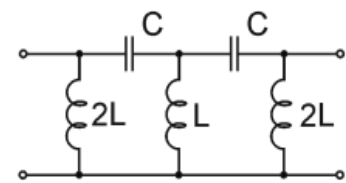

(a)

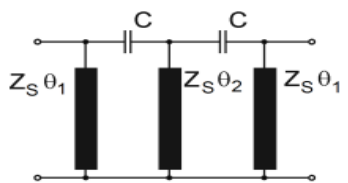

(b)
Fig. 3. Lumped element equivalent circuit of an LH transmission-line section (a) and its semi-distributed version (b).

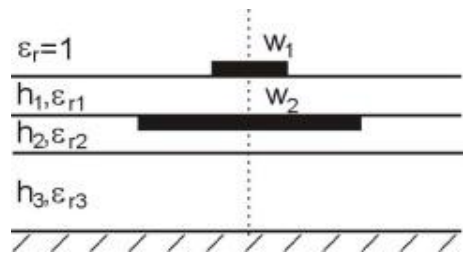

Fig. 4. Cross-section of the dielectric structure used for the design of the broadband balun circuit.

TABLE I: PARAMETERS OF THE COUPLED-LINE SECTION FOR WHICH $R=$ 1.43, DESIGNED IN THE STRUCTURE SHOWN IN Fig. 4, HAVING $h_{1}=0.152$ $\mathrm{mm}, \varepsilon_{r 1}=3.38, h_{2}=0.04 \mathrm{~mm}, \varepsilon_{r 2}=3.38, h_{3}=1.524 \mathrm{~mm}, \varepsilon_{r 3}=3.38$ AND $w_{1}=$ $0.92 \mathrm{~mm}, w_{2}=3.71 \mathrm{~mm}$

\begin{tabular}{llll}
\hline \hline Parameter & Value & Parameter & Value \\
\hline$C 11[\mathrm{pF} / \mathrm{m}]$ & 224.9 & $\mathrm{kC}$ & 0.81 \\
$C 22[\mathrm{pF} / \mathrm{m}]$ & 335.2 & $\mathrm{k}$ & 0.804 \\
$C m[\mathrm{pF} / \mathrm{m}]$ & 222.3 & $\mathrm{ZT} 1[\Omega]$ & 50 \\
$C 1[\mathrm{pF} / \mathrm{m}]$ & 2.6 & $\mathrm{ZT} 2,3[\Omega]$ & 35 \\
$C 2[\mathrm{pF} / \mathrm{m}]$ & 112.9 & $\mathrm{ZT} 4[\Omega]$ & 24.5 \\
$L 11[\mathrm{nH} / \mathrm{m}]$ & 395.7 & $\mathrm{ZL} 1[\Omega]$ & 41.95 \\
$L 22[\mathrm{nH} / \mathrm{m}]$ & 271.3 & $\mathrm{ZL} 2[\Omega]$ & 28.45 \\
$L m[\mathrm{nH} / \mathrm{m}]$ & 261.5 & $\varepsilon_{\text {effc }}$ & 2.972 \\
$k L$ & 0.789 & $\varepsilon_{\text {eff } \pi}$ & 2.759 \\
\hline \hline
\end{tabular}

To verify the properties of the proposed balun circuit, an electromagnetic calculation has been made in which the designed coupled-line section in conjunction with LH and RH transmission-line sections have been analyzed. The obtained $S$ parameters are presented in Fig. 5. One can notice a very good agreement between the circuit and electromagnetic analysis in terms of both amplitude and phase response. Finally, the proposed circuit has been fabricated and measured with the VNA under $50 \Omega$ terminating impedances at all ports. The obtained results have been recalculated to the proper terminating impedances at each port as denoted schematically in Fig. 1. The measurement results have been presented in Fig. 6, which fully confirm the results of both 
theoretical and electromagnetic analyses.

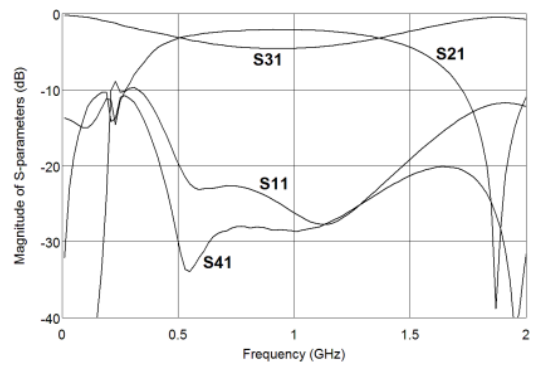

(a)

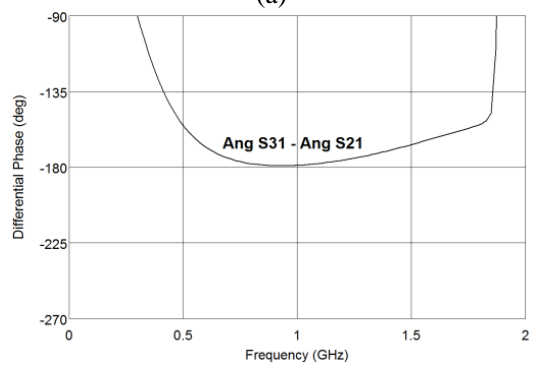

(b)

Fig. 5. Magnitude of $S$ parameters (a), and the differential phase (b) of the designed broadband balun circuit. Results of electromagnetic calculations.

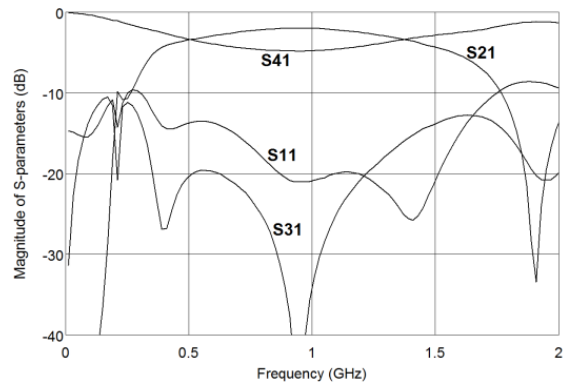

(a)

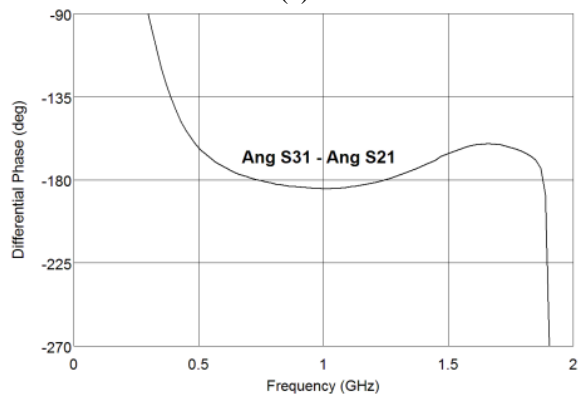

(b)

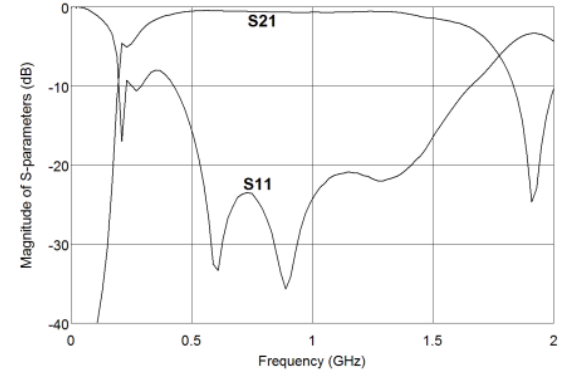

(c)

Fig. 6. Measured magnitude of $S$ parameters (a), the differential phase (b) of the designed broadband balun circuit and the $S$ parameters of its back-to-back connection (c).

From the measurement result of a single balun circuit, the properties of its back-to-back connection have been found, and the results are presented in Fig. 6(c). As it is seen the proposed circuit operates in over-an-octave frequency range with relatively low insertion losses and good impedance match. The photograph of the manufactured circuit is presented in Fig. 7.

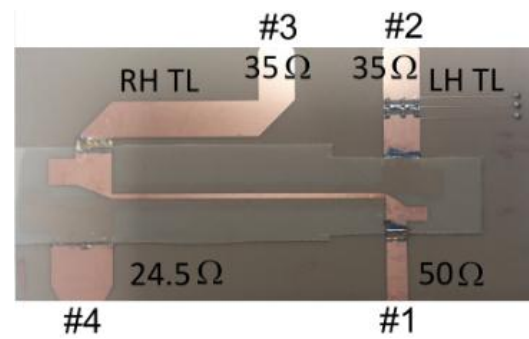

Fig. 7. A photo of the manufactured balun as a connection of the impedance transforming directional coupler with $45^{\circ}$-long $\mathrm{LH}$ and $\mathrm{RH}$ transmission-line sections.

\section{CONCLUSION}

In this paper, a novel approach to the design of the balun circuit has been presented in which an impedance transforming coupled-line section has been used in conjunction with an $\mathrm{LH} / \mathrm{RH}$ differential phase shifter. The proposed circuit allows for realization of baluns offering flexibility of impedance transformation ratio selection in a wide impedance range. The circuit features broadband frequency response exceeding one octave. The theoretical analyses have been confirmed with the measurement results of the designed balun.

\section{REFERENCES}

[1] Z. Y. Zhang and K. Wu, "A broadband substrate integrated waveguide SIW planar balun," IEEE Microwave and Wireless Components Letters, vol. 17, no. 12, pp. 843-845, Dec. 2007.

[2] N. Marchand, "Transmission line conversion transformers," Electronics, vol. 17, no. 12, pp. 142-145, Dec. 1944.

[3] K. S. Ang and I. D. Robertson, "Analysis and design of impedance-transforming planar Marchand baluns," IEEE Trans. Microw. Theory Tech., vol. 49, no. 2, pp. 402-406, Feb. 2001.

[4] H. R. Ahn and S. Nam, "New design formulas for impedance-transforming 3-dB Marchand baluns," IEEE Trans. Microw. Theory Tech., vol. 59, no. 11, pp. 2816-2823, Nov. 2011.

[5] C. I. Shie et al., "Design of a new type planar balun by using trans-directional couplers," IEEE Transactions on Microwave Theory and Techniques, vol. 60, no. 3, pp. 471-476, Mar. 2012.

[6] J. Sorocki, I. Piekarz, I. Slomian, S. Gruszczynksi, and K. Wincza "Single-layer coupled-line magic-Ts utilizing left-handed transmission line sections," in Proc. 20th International Conference on Microwaves, Radar, and Wireless Communication (MIKON), June 2014

[7] J. Sorocki, I. Piekarz, K. Wincza, and S. Gruszczynski, "Broadband magic-Ts with the use of coupled-line directional couplers and left-handed transmission line sections," Int. J. RF Microw. Comput.-Aided Eng., vol. 24, no. 4, Jul. 2014.

[8] K. Wincza and S. Gruszczynski, "Asymmetric coupled-line directional couplers as impedance transformers in balanced and $n$-way power amplifiers," IEEE Trans. Microw. Theory Techn., vol. 59, no. 7, pp. 1803-1810, Jul. 2011.

[9] A. Djordievic et al., Matrix Parameters for Multiconductor Transmission Lines, Software and User's Manual, Norwood, MA: Artech House, 1990.

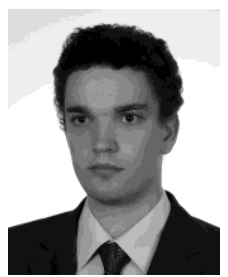

Jakub Sorocki received the M.Sc. degree in electronics and telecommunications from AGH University of Science and Technology, Cracow, Poland in 2013 and currently is working toward his $\mathrm{PhD}$ degree. Since 2011, he has been cooperating with Microwave Technology and High Frequency Electronics research team at the Department of Electronics, AGH UST. His scientific research interests focus on left-handed metamaterials in microwave range. He has coauthored few journals and conference papers. 


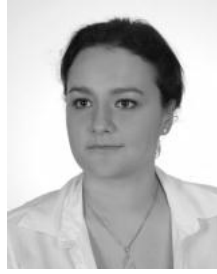

Ilona Piekarz received the M.Sc. degree in electronics and telecommunications from AGH University of Science and Technology, Cracow, Poland in 2013 and currently is working toward her $\mathrm{PhD}$ degree. Since 2011, she has been cooperating with Microwave Technology and High Frequency Electronics research team at the Department of Electronics, AGH UST. In 2013, she received "Diamond Grant" for outstanding students awarded by the Ministry of Science and Higher Education for her research on microwave biosensors. She has coauthored few journals and conference papers.

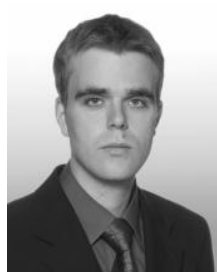

Kamil Staszek received his M.Sc. Tech. degree in electronics engineering from AGH University of Science and Technology, Cracow, Poland in 2011. He has coauthored 15 scientific papers. Currently he is working toward his Ph.D. degree at the same university in the field of microwave engineering, focusing on microwave measurement.

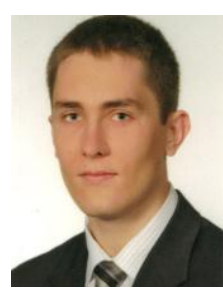

P. Kaminski received the B.Sc. degree in electronics and telecommunications from AGH University of Science and Technology, Cracow, Poland, in 2013. Since 2012, he has been cooperating with Microwave Technology and High Frequency Electronics research team at the Department of Electronics, AGH UST. His scientific interests lie in radar systems and their applications. He has coauthored 3 journals and 4 conference papers.

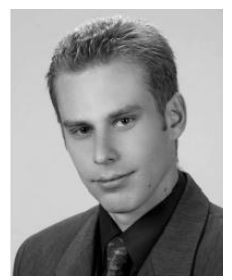

Krzysztof Wincza received the M.Sc. degree and the $\mathrm{Ph} . \mathrm{D}$. degree in electronics and electrical engineering from the Wroclaw University of Technology, Poland, in 2003 and 2007, respectively.

In 2007, he joined the Institute of Telecommunications, Teleinformatics and Acoustics, Wroclaw University of Technology. In 2009, he joined the Faculty of Electronics at AGH University of Science and Technology becoming an assistan

professor.

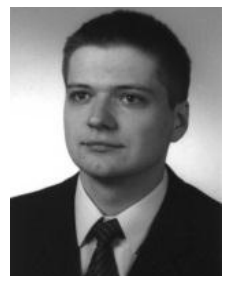

Slawomir Gruszczynski received the M.Sc. degree and the Ph.D. degree in electronics and electrical engineering from the Wroclaw University of Technology, Poland, in 2001 and 2006, respectively.

Since 2001 to 2006 , he has been with the Telecommunications Research Institute, Wroclaw Division. From 2005 to 2009, he worked at the Institute of Telecommunications, Teleinformatics and Acoustics, Wroclaw University of Technology. In 2009, he joined the Faculty of Informatics, Electronics and Telecommunications at AGH University of Science and Technology where he became a head of the Department of Electronics in 2012. He has coauthored 42 journal and 56 conference scientific papers. He is a member of the IEEE, and a member of Young Scientists' Academy at Polish Academy of Sciences (PAN) and Committee of Electronics and Telecommunications at Polish Academy of Sciences (PAN). 